\title{
SUNSPOTS WITH THE STRONGEST MAGNETIC FIELDS
}

\author{
W. LIVINGSTON, J.W. HARVEY, and O.V. MALANUSHENKO \\ National Solar Observatory, P.O. Box 26732, Tucson, AZ 85726, USA \\ (e-mail:wcl@nso.edu) \\ and \\ L. WEBSTER \\ Division of Astronomy and Astrophysics, University of California, Los Angeles, \\ CA 90095-1562, USA
}

(Received 11 July 2006; accepted 20 October 2006; Published online 5 December 2006)

\begin{abstract}
The strongest observed solar magnetic fields are found in sunspot umbrae and associated light bridges. We investigate systematic measurements of approximately 32000 sunspot groups observed from 1917 through 2004 using data from Mt. Wilson, Potsdam, Rome and Crimea observatories. Isolated observations from other observatories are also included. Corrections to Mt. Wilson measurements are required and applied. We found 55 groups $(0.2 \%)$ with at least one sunspot with one magnetic field measurement of at least $4000 \mathrm{G}$ including five measurements of at least $5000 \mathrm{G}$ and one spot with a record field of $6100 \mathrm{G}$. Although typical strong-field spots are large and show complex structure in white light, others are simple in form. Sometimes the strongest fields are in light bridges that separate opposite polarity umbras. The distribution of strongest measured fields above $3 \mathrm{kG}$ appears to be continuous, following a steep power law with exponent about -9.5 . The observed upper limit of 5-6 kG is consistent with the idea that an umbral field has a more or less coherent structure down to some depth and then fragments. We find that odd-numbered sunspot cycles usually contain about $30 \%$ more total sunspot groups but $60 \%$ fewer $>3 \mathrm{kG}$ spots than preceding even-numbered cycles.
\end{abstract}

\section{Introduction}

Sunspots are the most obvious manifestation of solar activity and yet our knowledge about them is still quite limited. Extreme examples of a phenomenon may help reveal its underlying physics. Here, we examine the distribution of the strongest magnetic fields measured in sunspots and what that may tell us about their origin and physics. A short survey of strong magnetic fields was done by Livingston (1974) and our interest in this topic was renewed by recent papers of V. G. Lozitsky and his colleagues (e.g. Lozitsky, 1993; Lozitsky and Staude, 2003), who hypothesize a role for 'superstrong' fields in connection with flares. He, and others, propose the existence of field strengths in excess of $5000 \mathrm{G}$ (even up to beyond $50000 \mathrm{G}$ ) which might occur in sub-telescopic (i.e. low filling factor) regions whose presence could only be deduced from multi-line profile observations with echelle-type spectrographs. Here, we do not pursue Lozitsky's ideas but simply inquire into what 
is known about strong fields from more conventional observations. What is the frequency of strong fields, do they occur in certain kinds of sunspots, what is their place within the activity cycle, and what physical conditions are necessary for their appearance?

Surveys of magnetic field strengths in sunspots have been published previously but usually without our emphasis on extreme values. (Following common practice, we use units of Gauss and the term field strength instead of flux density in this paper.) Ellerman (1919) commented that sunspot field strengths were typically around $2400 \mathrm{G}$ but could be as large as $4000 \mathrm{G}$. Nicholson (1933) studied the relationship between maximum field strengths and areas in sunspots and found a strong correlation for small areas that leveled off for large sunspots. Cowling (1946) confirmed this result and noted that visually measured field strengths showed a systematic decrease away from disk center, which he corrected in his analysis. von Klüber (1947) used different data and independently showed a correlation between field strength and area. Additional studies of this sort were done by Houtgast and van Sluiters (1948), Bell and Glazer (1958), and Ringes and Jensen (1960). A strong positive correlation between sunspot darkness and field strength was discovered by King (1934). The distribution function of the maximum magnetic field strengths of sunspot groups was discussed by Bell and Glazer (1959) and Bell (1959). They found that only about 5\% of 5940 sunspot groups had maximum measured fields greater than $3 \mathrm{kG}$. A thorough survey by Ringes (1965) extended this to 12293 groups from 1917 to 1958 and he found $4.3 \%$ of sunspot groups had maximum measured fields exceeding $3 \mathrm{kG}$ and $0.17 \%$ exceeding $4 \mathrm{kG}$.

\section{Measurements of Sunspot Field Strengths}

The Sun has been regularly mapped by photoelectric magnetographs since 1958 . These systems are designed to do a good job on weak fields but their fidelity fails in sunspot umbrae. This failure is basically because of scattered light, which mixes the spectra of polarized and unpolarized features. The recently retired Kitt Peak Vacuum Telescope magnetograph system, for example, seldom indicated fields above $2500 \mathrm{G}$. This same limitation applies to other magnetographs such as those at Mt. Wilson, the Crimea, and even the MDI SOHO instrument in space.

The first observations of magnetic fields in sunspots were made by photographing spectra using polarizing optics. At Mt. Wilson, irregular observations were made from 1908 through 1914 using both visual and photographic measurements of the wavelength separation between the Zeeman-split sigma components of simple triplets. Comparison with laboratory spectra produced under known magnetic field conditions allowed the wavelength splitting to be converted to field strength. After heroic efforts to detect a general magnetic field of the Sun, a systematic, 
daily program of sunspot field measurements was started in 1915 (Hale et al., 1919).

Visual measurements were pioneered by Seth Nicholson and involve the observer matching the two Zeeman sigma components of a simple triplet line such as Fe $617.33 \mathrm{~nm}$ with a tilting glass plate device (or parallel-plate micrometer). An image of the sunspot to be measured passes through a polarization analyzer placed in front of the slit of a spectrograph. The analyzer alternately transmits left and right circularly polarized light in narrow strips along the slit. The micrometer is placed at the focus of the spectrograph and, by tilt of the glass plate, shifts the wavelength position of the image of one strip until the two oppositely polarized Zeeman sigma components in adjacent strips coincide. The amount of shift corresponds to the field strength. Because of the width of spectrum lines, this method works poorly for fields below $1000 \mathrm{G}$ but becomes increasingly accurate above $2000 \mathrm{G}$. Stepanov, Shaposhnikova, and Petrova (1962) and Brants and Zwaan (1982) showed that scattered light degrades visual measurements of small sunspots at field strengths below about $2 \mathrm{kG}$. It is widely assumed that the Paschen-Back effect is not important for the spectrum lines used to measure the strongest umbral fields (von Klüber, 1955). We verified this assumption with a simple calculation.

At Mt. Wilson, Hale, and Nicholson (1938) initiated a program of measuring the magnetic fields in the prominent sunspots of all sunspot groups and noted these measurements on daily sunspot drawings. For each group a single maximum value was extracted which was published in a monograph (1917-1924), and starting in 1920 on a bi-monthly basis in the Publications of the Astronomical Society of the Pacific (PASP) (Nicholson, 1925-1960). In 1936, the observing program was changed from the daily measurement of each spot group to a minimum of one measurement per disk passage of a group (Anonymous, 1936), though in practice the measurements were usually done daily. The program continued under Nicholson's direction until his retirement in 1961. Thereafter, the program was carried on at Mt. Wilson by other observers including T. Cragg, and L. Webster. The priority of these observations was reduced in 1959 (Anonymous, 1958) and data collection and reduction procedures were altered in 1962 (Anonymous, 1962). These changes considerably reduced the quantity of the Mt. Wilson measurements and the regular program was suspended in September 2004.

Other observatories conducted similar observing programs and fortunately, just as Mt. Wilson eased off in 1961, Rome Observatory (Cimino, 1965-1975) took up the task and made observations for yet another decade and briefly in the mid-1980s. The Rome observing program is described in detail by Croce (1967). Their equipment is similar to Mt. Wilson. For our study, we mainly use the Mt. Wilson and Rome measurements. We also examined sunspot magnetic field measurements made at seven observatories in the former Soviet Union (http://www.gao.spb.ru/database/mfbase/) during the years 1957-1997. However, we were unable to integrate these measurements with the Mt. Wilson and Rome data without undertaking a major effort because spot groups were renumbered on a 
daily basis and cannot be tracked from day to day without recourse to the original observations. Photographic measurements were regularly made at Potsdam and we also examined their published results from 1946 to 1951 (Grotrian, 1953). The continuation of these results for 1954-1957 was published in a series of papers in volumes 283 and 284 of Astronomische Nachrichten. As far as we are aware, no systematic measurements optimized for sunspot magnetic field strengths are being done anywhere today.

Besides the above-extended campaigns, individual astronomers have taken photographic spectra showing exceptional field strengths. These include the remarkable isolated work of von Klüber in 1942 (von Klüber, 1947), the Crimean observations of Steshenko and colleagues (Baranovski and Petrova, 1957; Steshenko, 1967), Tanaka's (1991) Okayama observations, and our efforts at Kitt Peak (Livingston, 1976; Harvey, 1986).

In this paper, we examine the available data covering 1917-2004, mainly from Mt. Wilson and Rome, determine that corrections to the Mt. Wilson data are required, add the sporadic photographic measurements that confirm the reality of 4-6 kG fields, establish their temporal frequency, and inquire about the nature of the sunspots that produce them.

\subsection{MAXIMUM FIELD MEASUREMENTS: TECHNIQUE AND PROBLEMS}

Field strengths are deduced from Zeeman splitting. If the instrument has a circular polarization analyzer and the splitting is less than the spectral line width, it is sensitive to the longitudinal component of the field. Without an analyzer and with sufficient Zeeman splitting and spectral resolution the total field strength is measured.

It is now known that for typical sunspots the site of maximum field strength within an umbra is at the darkest, or coldest, position (Kopp and Rabin, 1992). (This fact was not used by visual observers who had no photometric means to set on the darkest place. As far as we can tell, the Mt. Wilson and Rome observers did not particularly search for the maximum field position. It was thought that field strength increased with umbral area (Nicholson, 1933).) Exceptions may be found in complex spots with light bridges that separate opposite polarity umbrae. Thus, the available historic data is very limited as a record of the strongest magnetic fields in sunspots. We can expect that many strong fields went unobserved simply because the observer did not know where to look.

Field strength measurements in the darkest and coldest parts of umbrae may be compromised by molecular blends close to the sigma components. In Table I, we list the spectrum lines most often employed. Inspecting the profiles of these lines in the spot spectral atlases of Wallace et al. (Wallace, Hinkle, and Livingston, 2001; Wallace and Livingston, 2005) we see blends in the coldest umbral examples. These are noted in the table. The column labeled $\sigma$ is the field strength that would place the sigma component on top of the blend. The last two columns are the 
TABLE I

Molecular blends of Fe I lines used for umbral field measurements.

\begin{tabular}{|c|c|c|c|c|}
\hline Line (nm) & Blends & $\sigma(\mathrm{kG})$ & $\chi(\mathrm{eV})$ & Zeeman pattern \\
\hline \multirow[t]{5}{*}{525.0217} & & & 0.13 & $(0) 3$ \\
\hline & $-0.022 \mathrm{FeH}$ & 5.7 & & \\
\hline & -0.013 & 3.4 & & \\
\hline & $+0.002 \mathrm{TiO}$ & 0.5 & & \\
\hline & $+0.025 \mathrm{FeH}$ & 6.5 & & \\
\hline \multirow[t]{6}{*}{617.3343} & & & 2.12 & (0) 2.5 \\
\hline & $-0.023 \mathrm{CaH}$ & 5.2 & & \\
\hline & -0.014 & 3.1 & & \\
\hline & +0.001 & 0.2 & & \\
\hline & +0.011 & 2.5 & & \\
\hline & $+0.032 \mathrm{TiO}$ & 7.2 & & \\
\hline \multirow[t]{4}{*}{630.2502} & & & 3.67 & (0) 2.5 \\
\hline & $-0.045 \mathrm{TiO}$ & 9.7 & & \\
\hline & -0.019 & 4.1 & & \\
\hline & $+0.041 \mathrm{TiO}$ & 7.2 & & \\
\hline \multirow[t]{6}{*}{1564.8518} & & & 5.43 & $(0) 3$ \\
\hline & $-0.092 \mathrm{CO}$ & 2.7 & & \\
\hline & -0.070 & 2.0 & & \\
\hline & +0.020 & 0.6 & & \\
\hline & $+0.068 \mathrm{CO}$ & 2.0 & & \\
\hline & +0.085 & 2.5 & & \\
\hline
\end{tabular}

excitation energy of the lower level of the transition that produces the spectrum line and its Zeeman splitting pattern expressed as $(\pi) \sigma$ in units of normal splitting. We do not know how observations might have been compromised by the presence of these blends. We note that the otherwise excellent line of Fe $1564.85 \mathrm{~nm}$ is badly contaminated by $\mathrm{CO}$ in dark umbrae.

Historical visual observations made with the parallel-plate micrometer cannot, of course, be verified except by comparison of near-simultaneous observations made at different observatories. We compared a few same day measurements from Mt. Wilson, Rome, Potsdam, Crimea and Pulkovo and generally found reasonably good agreement (within a few hundred Gauss; see also Steen and Maltby, 1959). In general, the observers were experienced and, we believe based on our personal acquaintance with some of them, conservative. If conditions were poor, say because of clouds or poor seeing, no field measurements were made and only polarities were recorded. One day in 1994, three experienced observers at Mt. Wilson each measured the same sunspot three times. This unique comparison showed consistency for a single observer of better than $10 \mathrm{G}$ and a range of $40 \mathrm{G}$ in the mean values 
between observers. At least in this case there is little indication of large differences in the measurements made by different observers.

Nicholson published the maximum field strengths for each spot group bimonthly in PASP. He did not elect to spell out the day of maximum. To get the exact day, we went to microfilm and scanned images of the original drawings. Even though the observer must have been excited, say by finding a value of $4200 \mathrm{G}$, there is no indication of this on the drawing; it was simply another measurement.

\subsection{CORRECTION OF MT. WILSON MEASUREMENTS}

Since the majority of available measurements comes from Mt. Wilson, we considered the method of measurement used there more closely. From 1917 through 1961, the observer wrote the tip angle of the parallel-plate micrometer in degrees on a drawing showing the sunspot group. The original construction and calibration of the micrometer were such that the tip of the glass plate in degrees corresponded approximately to the field strength in units of 100 Gauss using the $617.3 \mathrm{~nm}$ line. In compiling the published lists of measured field strengths, the nonlinear relationship between tip of a glass plate and image displacement was partly corrected by subtracting $1^{\circ}$ for values between 3 and $25^{\circ}$ and adding $1^{\circ}$ for values between 32 and $40^{\circ}$ (Hale and Nicholson, 1938). Thus, a reading of $25^{\circ}$ would become $2400 \mathrm{G}$ and $32^{\circ}$ would be $3300 \mathrm{G}$. This simple correction fails due to increasing nonlinearity at the large tilt angles needed to measure large field strengths. Therefore, we tried to reconstruct the calibration using available information.

The parallel-plate micrometer is still in use today after more than 90 years, but a few modifications have been made over the decades. For example, it now has two, nearly identical scales mounted together to indicate the tip of the glass plate in degrees. One scale is engraved in brass and the other one in ivory. We were unable to locate any records to indicate why the second scale was added. In any event, it does not significantly affect the measurements. The body of the micrometer was also modified at some point to allow it to be focussed. We examined old photographs of the micrometer to see when the modifications were made. It was in its original state in 1936 but had been modified by 1953 .

The original glass plate was removed from the micrometer in 1961 and replaced by a thicker one. Fortunately, the original plate has been located and measured. Its thickness is $4.0 \mathrm{~mm}$ and it appears to be a light crown glass with a presumed index of refraction of about 1.521 at $617.3 \mathrm{~nm}$. Using these values we verify that the micrometer measurements are accurate, using the simple corrections mentioned above, to within $100 \mathrm{G}$ from 0 to $35^{\circ}$ (or 0 to $3600 \mathrm{G}$ ). Larger tip angles produce underestimates. For example, $40^{\circ}$ corresponds to $4300 \mathrm{G}, 45^{\circ}$ to $5100 \mathrm{G}$ and $50^{\circ}$ to $5900 \mathrm{G}$. Thus, the observer might write 45 on the original drawing and this might have been published as $4600 \mathrm{G}$ while it would really correspond to $5100 \mathrm{G}$.

In comparing the original drawings with published measurements, we found that 
measurements of tip angles in excess of $40^{\circ}$ were not always consistently corrected by the nominal addition of $1^{\circ}$. Often, no correction was made.

The conversion of tip angle to field strength depends on the dispersion of the spectrograph and the known Landé $\mathrm{g}$ factor for the spectrum line. Here, we consider the history of changes of the value of the spectrograph reciprocal linear dispersion (also called plate factor). The Mt. Wilson spectrograph was originally equipped with a speculum grating ruled by Michelson. With a focal length of $22.9 \mathrm{~m}$, the reciprocal dispersion was quoted as $2.96 \mathrm{~mm}^{-1}$ for the $6173 \AA$ line in second order (Hale et al., 1919) and $4.9 \mathrm{~mm}^{-1}$ at $5900 \AA$ in the third order (Hale and Nicholson, 1938). These values are consistent with a grating spacing of 602 grooves $\mathrm{mm}^{-1}$ but not with the published spacing of 622 . We believe that the latter number is a typographical error and adopt the 602 value. In 1930, a grating (9A) was ruled by Mt. Wilson Observatory with 602 grooves $\mathrm{mm}^{-1}$ and was used thereafter interchangeably with the Michelson grating with no change in dispersion. In May 1949, a new grating (27B) having 400 grooves $\mathrm{mm}^{-1}$ was installed (Richardson and Schwarzschild, 1950). This change decreased the second-order reciprocal dispersion by a large amount. However, the third-order dispersion was virtually identical with that of the previous gratings used in second order and we presume that the observers therefore used third order to maintain continuity. This grating was replaced with another 400 grooves $\mathrm{mm}^{-1}$ one (33B) on September 29, 1950. On May 19, 1955, a 600 grooves $\mathrm{mm}^{-1}$ grating (91B) was installed followed by a similar one (118B) on August 22, 1960. A 610 grooves $\mathrm{mm}^{-1}$ (180Bic) was installed on December 24, 1962 and was used until the spectrograph was flooded during a severe storm in 1978 . With some degradation this grating continued to be used until a Bausch and Lomb grating with 632 grooves $\mathrm{mm}^{-1}$ was installed on May 17, 1982. On November 21, 1994 a Milton-Roy grating with 367.5 grooves $\mathrm{mm}^{-1}$ was installed and remains in place today. Thus, remarkably for the sunspot magnetic field measurements, the reciprocal linear dispersion of the spectrograph was little changed for 80 years from 1914 until 1994.

In October 1961, the use of the $617.3 \mathrm{~nm}$ line was terminated in favor of the $525.0 \mathrm{~nm}$ line in the fifth order in order to accommodate increasing use of the photoelectric magnetograph. The glass plate in the micrometer was replaced with one of $7 \mathrm{~mm}$ thickness at that time. With the much larger spectrograph reciprocal linear dispersion, the conversion recipe of angle of the parallel plate to field strength was changed. The tip angle was divided by two and the resulting odd half-degree angles were rounded up to the next full degree. Thus, $45^{\circ}$ was called 23 on the drawing or $2300 \mathrm{G}$. We checked this recipe and found that it works within $100 \mathrm{G}$ up to tip angles of $32^{\circ}$ which corresponds to an actual field strength of $1680 \mathrm{G}$. At larger angles, the nonlinear behavior of displacement versus tip angle produces serious underestimates of the field strength. For example, a tip of $60^{\circ}$ (the limit of the calibrated scale on the micrometer) would be recorded as $3000 \mathrm{G}$ but is actually $3880 \mathrm{G}$. The recipe changed again in 1994 when the grating was replaced. At that time, up until regular observations were suspended, observers used a table 
to convert tip angle into field strength in Gauss. We checked the values and find the table to be correct at $2200 \mathrm{G}$ but to overestimate true field strength at smaller tip angles and underestimate it at larger tips or field strengths. For example, at the large tip angle of $60^{\circ}$, the table indicates $2600 \mathrm{G}$ while the true strength is $2960 \mathrm{G}$.

Table II shows corrections that we applied to the Mt. Wilson measurements based on what we learned about the parallel-plate micrometer and spectrograph dispersion. Note that the strongest measurable field strength at Mt. Wilson was in excess of $6000 \mathrm{G}$ until 1961, $4000 \mathrm{G}$ after 1961 and only $3000 \mathrm{G}$ after 1994.

TABLE II

Corrections to Mt. Wilson measurements (units of $100 \mathrm{G}$ ).

\begin{tabular}{|c|c|c|c|c|}
\hline \multirow[b]{2}{*}{ Drawing value } & \multirow{2}{*}{$\begin{array}{l}\text { Published field } \\
1917-1961\end{array}$} & \multicolumn{3}{|c|}{ True field } \\
\hline & & $1917-1961$ & $1961-1994$ & $1994-2004$ \\
\hline 25 & 25 & 24 & 28 & 27 \\
\hline 26 & 26 & 25 & 30 & 29 \\
\hline 27 & 27 & 27 & 32 & 31 \\
\hline 28 & 28 & 28 & 34 & \\
\hline 29 & 29 & 29 & 36 & \\
\hline 30 & 30 & 30 & 38 & \\
\hline 31 & 31 & 31 & 40 & \\
\hline 32 & 33 & 33 & & \\
\hline 33 & 34 & 34 & & \\
\hline 34 & 35 & 35 & & \\
\hline 35 & 36 & 36 & & \\
\hline 36 & 37 & 38 & & \\
\hline 37 & 38 & 39 & & \\
\hline 38 & 39 & 41 & & \\
\hline 39 & 40 & 42 & & \\
\hline 40 & 41 & 43 & & \\
\hline 41 & 42 & 45 & & \\
\hline 42 & 43 & 46 & & \\
\hline 43 & 44 & 48 & & \\
\hline 44 & 45 & 49 & & \\
\hline 45 & 46 & 51 & & \\
\hline 46 & 47 & 53 & & \\
\hline 47 & 48 & 54 & & \\
\hline 48 & 49 & 56 & & \\
\hline 49 & 50 & 58 & & \\
\hline 50 & 51 & 59 & & \\
\hline 51 & 52 & 61 & & \\
\hline 52 & 53 & 63 & & \\
\hline
\end{tabular}




\section{Observations}

We began listing sunspots with exceptionally strong magnetic fields by first making a list of the year, month and strongest field measured in each sunspot group from Mt. Wilson publications. For the years 1917 through 1924, the observations are from Hale and Nicholson (1938). For 1925-1959, we used the tables published bi-monthly by Nicholson (Nicholson, 1925-1960). For 1959 and 1960, we had access to a copy of the Mt. Wilson listing of sunspot groups that tabulated the field strengths. For the years 1961 through 1964, we used microfilm copies of the Mt. Wilson drawings. In nearly all cases when a strong-field measurement was published, we were able to check it by consulting either a microfilm or an online copy of the original drawing, and corrections were applied as needed to the measurements. We used Rome data from Solar Phenomena Monthly Bulletins (Cimino, 1965 - 1975) to extend the list from 1964 to 1974 (and for a brief period in the mid-1980s). No corrections were made to the Rome data. We also systematically searched the published 1946-1957 Potsdam data for strong-field measurements. Later, a digitized listing of Mt. Wilson measurements from 1915 to 2002, compiled by T. Shieber, was located in the Mt. Wilson Observatory archives. This listing records the maximum magnetic field strength only in $500 \mathrm{G}$ categories as integers ranging from 1 to 9 . A search of it identified spot groups with potentially strong fields and we then looked at the original drawings for more detail. In a second step, we added various published reports of strong magnetic field measurements from literature known to us.

Next, the preliminary list of strong reported fields was augmented with nearly simultaneous measurements from other observatories when possible. These additional measurements were generally less strong and we may attribute the difference either to the length of time between the measurements or to differences in the locations of the measurements within the spot groups.

Table III lists in chronological order the strong-field measurements that have come to our attention. The measurements are believed to be photographic in all cases except those of Mt. Wilson and Rome, which were done visually. We are not certain if the routine Crimea measurements were done visually or photographically.

The large number of measurements in this table by J. Hickox is simply because he was the principal solar observer at Mt. Wilson from 1921 to 1923 and 1928 to 1961.

Entry 52 is included since it is possible this spot reached the $4 \mathrm{kG}$ limit.

For a few entries in Table III, there are two measurements for the same day from the same observatory (e.g. entry 2). These indicate two different sunspots in the same group with field strengths in excess of $4 \mathrm{kG}$.

Concerning the $4900 \mathrm{G}$ field recorded by Baranovsky and Petrova (1957) (entry 38), N. Stepanian has loaned us the original plates which are reproduced in part in Figure 1. Our remeasurement yields about $5000 \mathrm{G}$, confirming their results. Interestingly, the routine daily measurements from Crimea for this sunspot 
TABLE III

Sunspot groups with at least one measured magnetic field $\geq 4000 \mathrm{G}$.

\begin{tabular}{|c|c|c|c|c|c|c|c|}
\hline$N$ & Group & Date & $B(\mathrm{G})$ & Lat & Obs & Observer & Ref \\
\hline 1 & 1402 & 1919 Apr 1 & 4000 & -20 & M & Rodés & 1 \\
\hline \multirow[t]{2}{*}{2} & 2095 & 1924 Aug 30 & 4200 & 22 & M & Humason & 1,4 \\
\hline & $"$ & $"$ & 4600 & $"$ & M & $"$ & 1,4 \\
\hline 3 & 3726 & 1929 Jun 24 & 4300 & -10 & M & Hickox & 2 \\
\hline 4 & 3754 & 1929 Jul 16 & 4600 & -17 & M & Richardson & 2 \\
\hline 5 & 5548 & 1937 Sep 9 & 4600 & 11 & M & Hickox & 2,3 \\
\hline 6 & 6192 & 1938 Nov 8 & 4200 & -9 & M & $"$ & 2,3 \\
\hline 7 & 6725 & 1940 Jan 5 & 4100 & 12 & M & $"$ & 2,3 \\
\hline 8 & 7297 & 1941 Oct 1 & 4100 & 2 & M & $"$ & 3 \\
\hline 9 & 7315 & 1941 Oct 31 & 4200 & 14 & M & $"$ & 2,3 \\
\hline \multirow[t]{5}{*}{10} & 7378 & 1942 Feb 28 & 6100 & 8 & M & $"$ & 2,3 \\
\hline & $"$ & 1942 Mar 2 & 5900 & $”$ & M & $"$ & 3 \\
\hline & $"$ & $"$ & 4800 & $"$ & M & $"$ & 3 \\
\hline & $"$ & 1942 Mar 3 & 4300 & $"$ & $\mathrm{P}$ & Haffner & 5 \\
\hline & $"$ & 1942 Mar 4 & 4500 & $"$ & M & Hickox & 3 \\
\hline \multirow[t]{2}{*}{11} & 7550 & 1943 Feb 10 & 4500 & 5 & M & $"$ & 2,3 \\
\hline & $"$ & $1943 \mathrm{Feb} 11$ & 4200 & $"$ & M & $"$ & 3 \\
\hline 12 & 7569 & 1943 Apr 3 & 4200 & 33 & M & $"$ & 2,3 \\
\hline 13 & 7617 & 1943 Oct 3 & 4300 & 16 & M & $"$ & 2,3 \\
\hline 14 & 7851 & 1945 Oct 4 & 4300 & -22 & M & $"$ & 2,3 \\
\hline 15 & 8170 & 1946 Aug 29, 31 & 4200 & 9 & M & $"$ & 2,3 \\
\hline \multirow[t]{2}{*}{16} & 8319 & 1946 Dec 14,16 & 4900 & -6 & M & $"$ & 2,3 \\
\hline & $"$ & 1946 Dec 18 & 4300 & $"$ & M & Richardson & 3 \\
\hline \multirow[t]{4}{*}{17} & 8707 & 1947 Jul 15 & 4300 & 13 & M & $"$ & $2,3,4$ \\
\hline & $"$ & 1947 Jul 16 & 4100 & $"$ & M & Hickox & 3 \\
\hline & $"$ & 1947 Jul 17 & 3700 & $"$ & $\mathrm{P}$ & & 6 \\
\hline & $"$ & 1947 Jul 18 & 4100 & $"$ & M & Hickox & 3 \\
\hline 18 & 8774 & 1947 Aug 15 & 4300 & 15 & M & Richardson & $2,3,4$ \\
\hline \multirow[t]{2}{*}{19} & 8833 & 1947 Sep 27 & 4200 & 17 & M & Hickox & $2,3,4$ \\
\hline & $"$ & 1947 Sep 26 & 3000 & $"$ & $\mathrm{P}$ & & 6 \\
\hline \multirow[t]{2}{*}{20} & 9086 & 1948 Mar 4 & 4200 & 17 & M & Hickox & 2,3 \\
\hline & $"$ & $"$ & 3200 & $"$ & $\mathrm{P}$ & & 6 \\
\hline \multirow[t]{2}{*}{21} & 9150 & 1948 Apr 16, 17 & 4200 & 9 & M & Hickox & 2,3 \\
\hline & $"$ & 1948 Apr 17 & 3400 & $"$ & $\mathrm{P}$ & & 6 \\
\hline \multirow[t]{2}{*}{22} & 9167 & 1948 Apr 26 & 4300 & 23 & M & Hoge & 2,3 \\
\hline & $"$ & 1948 Apr 25 & 2400 & $"$ & $\mathrm{P}$ & & 6 \\
\hline
\end{tabular}


TABLE III

(Continued).

\begin{tabular}{|c|c|c|c|c|c|c|c|}
\hline$N$ & Group & Date & $B(\mathrm{G})$ & Lat & Obs & Observer & Ref \\
\hline \multirow[t]{3}{*}{23} & 9199 & 1948 May 10 & 4200 & -24 & M & Hickox & 2,3 \\
\hline & $"$ & 1948 May 12 & 4100 & $"$ & M & $"$ & 3 \\
\hline & ” & $"$ & 3200 & $"$ & $\mathrm{P}$ & & 6 \\
\hline \multirow[t]{2}{*}{24} & 9275 & 1948 Jun 28 & 4200 & 12 & M & Hickox & 2,3 \\
\hline & $"$ & 1948 Jun 30 & 4100 & $”$ & M & $"$ & 3 \\
\hline \multirow[t]{2}{*}{25} & 9317 & 1948 Jul 22-29 & 4300 & 12 & M & Richardson & 2,3 \\
\hline & $"$ & 1948 Jul 26 & 2800 & $"$ & $\mathrm{P}$ & & 6 \\
\hline \multirow[t]{2}{*}{26} & 10027 & 1949 Oct 5,8 & 4200 & 7 & M & Hickox & 2,3 \\
\hline & $"$ & 1949 Oct 8 & 2800 & $"$ & $\mathrm{P}$ & & 6 \\
\hline 27 & 10087 & 1949 Nov 6 & 4300 & -10 & M & Hickox & 2,3 \\
\hline \multirow[t]{2}{*}{28} & 10262 & 1950 Mar 14 & 4300 & -16 & M & $"$ & 2,3 \\
\hline & $"$ & $"$ & 2700 & $"$ & $\mathrm{P}$ & & 6 \\
\hline \multirow[t]{2}{*}{29} & 10303 & 1950 Apr 13 & 4600 & 13 & M & Roques & 2,3 \\
\hline & $"$ & $"$ & 2500 & $"$ & $\mathrm{P}$ & & 6 \\
\hline \multirow[t]{4}{*}{30} & 10314 & 1950 Apr 25, 30 & 4200 & -12 & M & Hickox & 2,3 \\
\hline & $"$ & 1950 Apr 26 & 2800 & $"$ & $\mathrm{P}$ & & 6 \\
\hline & $"$ & 1950 Apr 27 & 4300 & $”$ & M & Hickox & 2,3 \\
\hline & $"$ & 1950 Apr 29 & 4100 & $"$ & M & $"$ & 2,3 \\
\hline 31 & 10347 & 1950 May 20 & 4300 & -10 & M & $"$ & 2,3 \\
\hline 32 & 10382 & 1950 Jun 22,23 & 4600 & 9 & M & Roques & 2,3 \\
\hline 33 & 10384 & 1950 Jun 26 & 4600 & 9 & M & $"$ & 2,3 \\
\hline 34 & 11352 & 1955 Oct 24 & 4100 & 28 & M & Richardson & 2,3 \\
\hline 35 & 11353 & 1955 Oct 24 & 4100 & -23 & M & $"$ & 2,3 \\
\hline 36 & 11466 & 1956 Feb 20 & 4300 & 21 & M & Cragg & 2,3 \\
\hline 37 & 12417 & 1957 Jun 18 & 4100 & 18 & M & 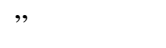 & 2,3 \\
\hline \multirow[t]{8}{*}{38} & 12491 & 1957 Jul 17 & 5000 & 28 & $\mathrm{C}$ & & 7 \\
\hline & 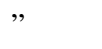 & $"$ & 4900 & $"$ & $\mathrm{C}$ & & 8 \\
\hline & $"$ & $"$ & 2000 & $"$ & M & Hickox & 3,4 \\
\hline & $"$ & 1957 Jul 18 & 4900 & $"$ & $\mathrm{C}$ & & 8 \\
\hline & 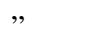 & $"$ & 4600 & 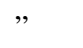 & $\mathrm{C}$ & & 7 \\
\hline & $"$ & 1957 Jul 19 & 4700 & $"$ & $\mathrm{C}$ & & 7 \\
\hline & $"$ & $"$ & 4400 & $"$ & $\mathrm{C}$ & & 8 \\
\hline & $"$ & $"$ & 2300 & $"$ & M & Hickox & 3,4 \\
\hline 39 & 13388 & $1958 \mathrm{Jul} 30$ & 5350 & -15 & $\mathrm{C}$ & SS & 9 \\
\hline 40 & 15733 & 1963 Jun 14 & 4000 & 11 & $\mathrm{C}$ & & 7 \\
\hline \multirow[t]{2}{*}{41} & 16150 & 1966 Oct 15 & $>4000$ & 7 & M & Cragg & 3 \\
\hline & 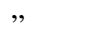 & 1966 Oct 19 & 4300 & $"$ & $\mathrm{R}$ & $\mathrm{CCF}$ & 10 \\
\hline
\end{tabular}


TABLE III

(Continued).

\begin{tabular}{|c|c|c|c|c|c|c|c|}
\hline$N$ & Group & Date & $B(\mathrm{G})$ & Lat & Obs & Observer & Ref \\
\hline \multirow[t]{4}{*}{42} & 16387 & 1967 Jun 4 & 3600 & 23 & M & Cragg & 3 \\
\hline & $"$ & 1967 Jun 5 & 4000 & $"$ & $\mathrm{R}$ & $\mathrm{CCF}$ & 10 \\
\hline & $"$ & $"$ & 2800 & $"$ & $\mathrm{C}$ & & 7 \\
\hline & $"$ & $"$ & 3000 & $"$ & M & Cragg & 3 \\
\hline \multirow[t]{4}{*}{43} & 17196 & 1969 Mar 20 & 3600 & 22 & M & Utter & 3 \\
\hline & $"$ & 1969 Mar 22 & 4000 & $"$ & $\mathrm{R}$ & $\mathrm{CCF}$ & 10 \\
\hline & $"$ & $"$ & 2500 & $"$ & $\mathrm{C}$ & & 7 \\
\hline & $"$ & 1969 Mar 23 & 3000 & $"$ & M & Utter & 3 \\
\hline \multirow[t]{7}{*}{44} & 17822 & 1970 Apr 7 & 4000 & -12 & M & Cragg & 3 \\
\hline & $"$ & 1970 Apr 8 & 4300 & $"$ & $\mathrm{R}$ & $\mathrm{CCF}$ & 10 \\
\hline & $"$ & $"$ & 3100 & $"$ & $\mathrm{C}$ & & 7 \\
\hline & $"$ & $"$ & 3400 & $"$ & M & Cragg & 3 \\
\hline & $"$ & 1970 Apr 9 & 3800 & $"$ & M & $"$ & 3 \\
\hline & $"$ & 1970 Apr 10 & 3600 & $"$ & M & $"$ & 3 \\
\hline & $"$ & 1970 Apr 11 & 3800 & $"$ & M & $"$ & 3 \\
\hline \multirow[t]{8}{*}{45} & 18935 & 1972 Jul 31 & 3400 & 12 & M & $"$ & 3 \\
\hline & $"$ & 1972 Aug 5 & 3200 & $"$ & M & $"$ & 3 \\
\hline & $"$ & 1972 Aug 7 & 4100 & $"$ & $\mathrm{R}$ & $\mathrm{CF}$ & 10 \\
\hline & $"$ & $"$ & 3100 & $"$ & $\mathrm{C}$ & & 7 \\
\hline & 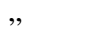 & 1972 Aug 8 & 5150 & $"$ & $\mathrm{~K}$ & & 11 \\
\hline & $"$ & $"$ & 2600 & $"$ & $\mathrm{C}$ & Shpitalnaya & 7 \\
\hline & $"$ & $"$ & 3000 & $"$ & $\mathrm{R}$ & $\mathrm{CF}$ & 10 \\
\hline & 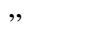 & $"$ & 2500 & $"$ & M & Cragg & 3 \\
\hline \multirow[t]{5}{*}{46} & 19427 & 1974 Jul 3 & 4300 & -14 & $\mathrm{O}$ & Tanaka & 17 \\
\hline & $"$ & 1974 Jul 4 & 4200 & $"$ & $\mathrm{C}$ & & 7 \\
\hline & $"$ & $"$ & 3200 & $"$ & $\mathrm{R}$ & $\mathrm{CC}$ & 10 \\
\hline & $"$ & $"$ & 4130 & $"$ & $\mathrm{~N}$ & Livingston & 12 \\
\hline & $"$ & $"$ & 3600 & $"$ & M & Cragg & 3 \\
\hline \multirow[t]{6}{*}{47} & 19469 & 1974 Sep 11 & 4000 & 8 & $\mathrm{R}$ & $\mathrm{CC}$ & 10 \\
\hline & $”$ & $"$ & 3200 & $”$ & $\mathrm{C}$ & & 7 \\
\hline & $"$ & $"$ & 3200 & $"$ & M & Cragg & 3 \\
\hline & $"$ & 1974 Sep 13 & 4000 & $"$ & $\mathrm{R}$ & $\mathrm{CC}$ & 10 \\
\hline & $"$ & $"$ & 3300 & $"$ & $\mathrm{C}$ & & 7 \\
\hline & $"$ & " & 3200 & $"$ & M & Cragg & 3 \\
\hline \multirow[t]{4}{*}{48} & 19512 & 1974 Nov 19 & 4100 & 14 & $\mathrm{C}$ & & 7 \\
\hline & $"$ & $"$ & 3800 & $"$ & $\mathrm{R}$ & $\mathrm{CC}$ & 10 \\
\hline & $"$ & 1974 Nov 21 & 3200 & $"$ & M & Adkins & 3 \\
\hline & & & & & \multicolumn{3}{|c|}{ (Continued on next page) } \\
\hline
\end{tabular}


TABLE III

(Continued).

\begin{tabular}{|c|c|c|c|c|c|c|c|}
\hline$N$ & Group & Date & $B(\mathrm{G})$ & Lat & Obs & Observer & Ref \\
\hline \multirow[t]{5}{*}{49} & 20123 & 1978 Jul 13 & 4300 & 18 & $\mathrm{~N}$ & Harvey & 13 \\
\hline & $"$ & $"$ & 3600 & $"$ & $\mathrm{C}$ & & 7 \\
\hline & $"$ & $”$ & 2700 & $"$ & M & Gregory & 3 \\
\hline & $”$ & 1978 Jul 14 & 3800 & $"$ & $\mathrm{C}$ & & 7 \\
\hline & 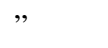 & $"$ & 2500 & $"$ & M & Gregory & 3 \\
\hline \multirow[t]{3}{*}{50} & 21358 & 1980 Apr 8 & 3200 & 13 & M & Webster & 3 \\
\hline & $"$ & 1980 Apr 9 & 4000 & $"$ & $\mathrm{C}$ & & 14 \\
\hline & $"$ & $"$ & 2800 & $"$ & M & Webster & 3 \\
\hline \multirow[t]{4}{*}{51} & 21567 & 1980 Jul 6 & 4100 & 28 & $\mathrm{C}$ & & 14 \\
\hline & $"$ & $"$ & 2100 & $"$ & M & Webster & 3 \\
\hline & $"$ & $1980 \mathrm{Jul} 7$ & 4600 & 28 & $\mathrm{C}$ & & 14 \\
\hline & ” & $"$ & 2100 & $"$ & M & Webster & 3 \\
\hline \multirow[t]{2}{*}{52} & 24223 & 1985 May 11 & $>3800$ & -12 & $\mathrm{R}$ & Croce & 10 \\
\hline & $"$ & 1985 May 13 & 3500 & $"$ & $\mathrm{C}$ & & 7 \\
\hline \multirow[t]{2}{*}{53} & 26825 & 1991 Jun 6 & 3800 & 32 & M & $?$ & 4 \\
\hline & $"$ & 1991 Jun 7 & 4100 & $"$ & $\mathrm{C}$ & & 7,14 \\
\hline \multirow[t]{2}{*}{54} & 31909 & 2003 Oct 22 & 4200 & 4 & $\mathrm{C}$ & Stepanian & 15 \\
\hline & $"$ & $"$ & $>3000$ & $"$ & M & Padilla & 4 \\
\hline \multirow[t]{2}{*}{55} & 32158 & 2004 Jul 23 & 4100 & 8 & $\mathrm{C}$ & & 16 \\
\hline & $"$ & $"$ & $>3000$ & $"$ & M & Gilman & 4 \\
\hline
\end{tabular}

Notes. Group is the Mt. Wilson Observatory sunspot group number. $B(\mathrm{G})$ is the magnetic field strength in Gauss. Lat is the latitude in degrees. Obs is the observatory abbreviated as follows: $\mathrm{C}=$ Crimean Astrophysical Obs., $\mathrm{K}=$ Kislovodsk, $\mathrm{M}=$ Mt. Wilson Obs., $\mathrm{N}=$ National Solar Obs., Kitt Peak, $\mathrm{O}=$ Okayama, $\mathrm{P}=$ Potsdam, $\mathrm{R}=$ Astro. Obs. of Rome. Observers are abbreviated as follows: $\mathrm{CC}=$ Casamassima and Croce, $\mathrm{CCF}=$ Cacciani, Croce and Flamini, $\mathrm{CF}=$ Croce and Flamini, SS = Stepanian and Selivanov. References: 1. Hale and Nicholson (1938). 2. Pub. Astro. Soc. Pacific, bi-monthly from 1920 to 1961. 3. Microfilm copies of original Mt. Wilson drawings. 4. ftp://howard.astro.ucla.edu/ pub/obs/drawings/. 5. von Klüber (1947). 6. Pub. Astro. Obs. Potsdam. 7. http://www.gao.spb.ru/ database/mfbase/gmaps/krao/. 8. Baranovsky and Petrova (1957). 9. Steshenko (1967). 10. Solar Phenomena, Astro. Obs. Rome, monthly. 11. Shpitalnaya, Makarov and Den (1973). 12. Livingston (1974). 13. Harvey (1986). 14. Solar Data Bulletin, Pulkovo Obs. 15. Personal communication. 16. http://crao.crimea.ua/projects/solar/ sunspot/DATA/. 17. Tanaka (1991).

show 5000, 4600 and $4700 \mathrm{G}$ for July 17, 18, 19, respectively. At Mt. Wilson the same spot was recorded as 2000 and $2300 \mathrm{G}$ on July 17 and 19, respectively. Perhaps the strong fields were confined to a part of the sunspot that was not measured at Mt. Wilson. Figure 2 suggests that this might be the case. 


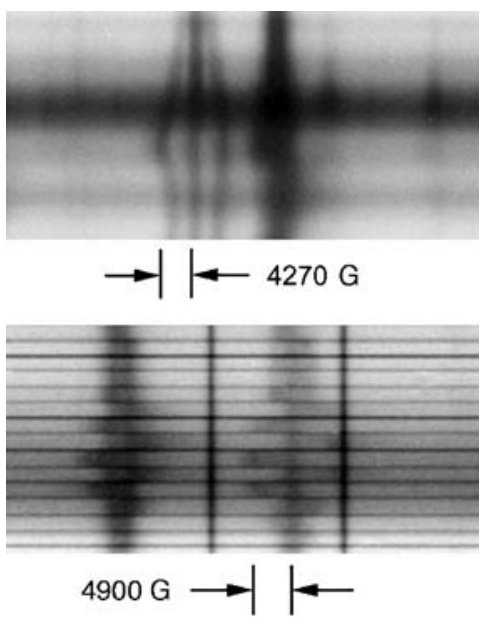

Figure 1. Examples of spectra. Top was obtained by J. Harvey (1986) using $525.0 \mathrm{~nm}$ on July 13, 1978 and refers to a light bridge in a complex sunspot. Bottom is from Baranovsky and Petrova (1957) using $630.2 \mathrm{~nm}$ on July 18, 1957. Field strengths are based on the total separation of the sigma components.

Also shown in Figure 1 is J. Harvey's spectrum of July 13, 1978 (entry 49) which shows a $4300 \mathrm{G}$ field based on the separation of the sigma components. There is a strong local Doppler shift at the location of the strongest field. This sunspot was measured visually at Crimea some hours earlier as having a $3600 \mathrm{G}$ field in the umbra. It was not measured at Mt. Wilson. Photographs exposed to show the umbral intensity indicate it was darkest adjacent to the light bridge in which the strong field was detected.

The Kislovodsk observation (entry 45) of Shpitalnaya et al. (Shpitalnaya, Makarov, and Den, 1973) is unique in that the field was recorded in 14 different Fe lines simultaneously. Values ranged from 4830 to $5300 \mathrm{G}$ from which they conclude a field of $5150 \pm 150 \mathrm{G}$. The authors also include white light, $\mathrm{H} \alpha$, and $\mathrm{Ca} \mathrm{K}$ pictures of the region, with the position of interest found abutting a light bridge.

We compared the Potsdam and Mt. Wilson observations for the three 1950 sunspots in Table III. The measurements of all the spots on the disk agreed to within a few hundred Gauss except for the three strong-field spots. The Potsdam measurements were about half the field strengths measured at Mt. Wilson. However, Potsdam measurements were generally systematically smaller than Mt. Wilson at high field strengths, which Grotrian (1953) attributed to the use of different spectral lines. We think that this explanation is unlikely. On the other hand, the largest strength measured during 1946-1951 at Potsdam was 3600 G on July 13, 1951. Mt. Wilson measurements of this sunspot gave essentially the same field strength. These conflicting results indicate either that the three 1950 measurements from Mt. Wilson or Potsdam were wrong or that the sunspots changed rapidly. It is also possible that different parts of the sunspots were measured at the two observatories. 

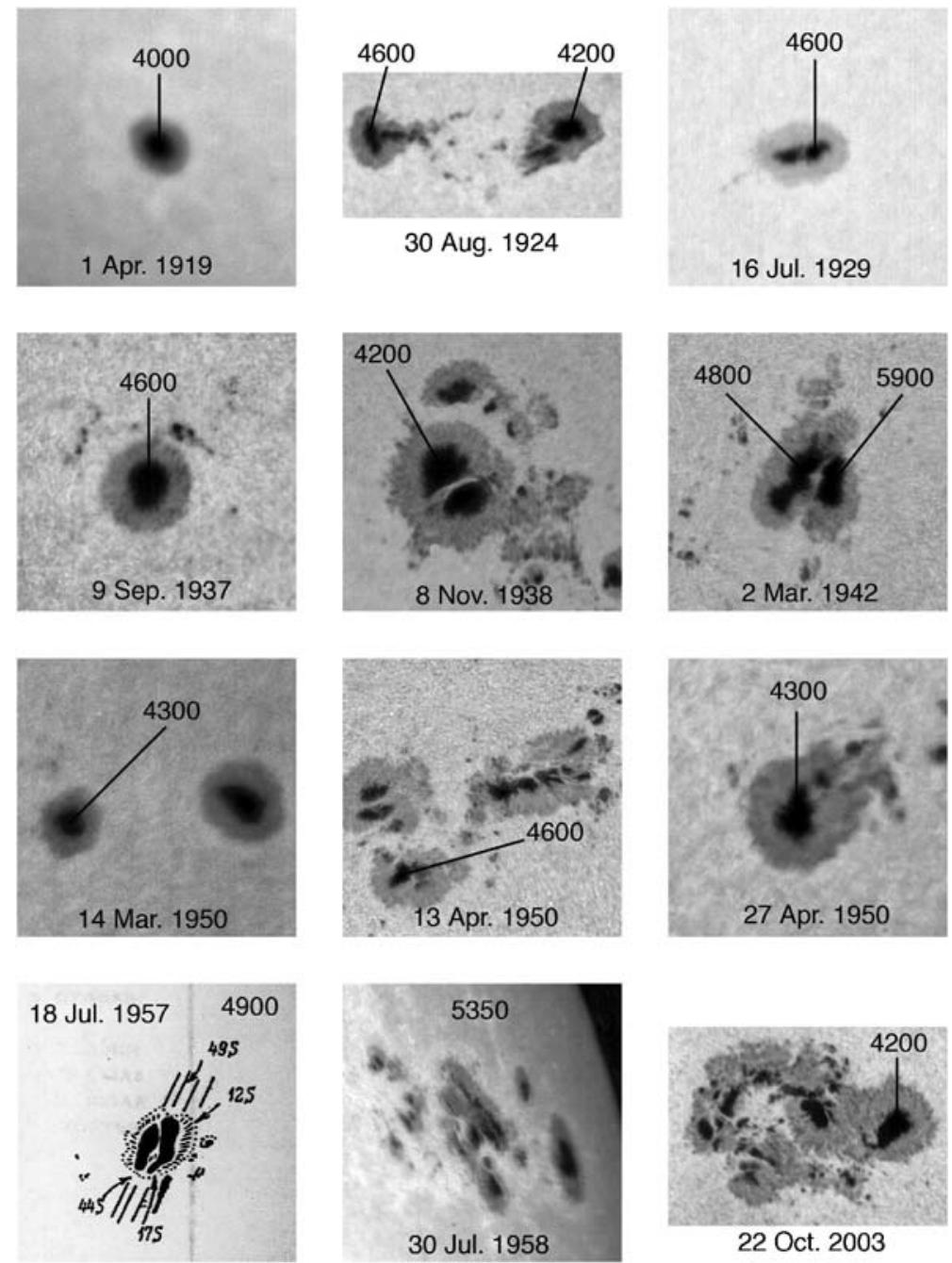

Figure 2. White light photographs (and a drawing) of strong-field spots. The width of the photographic panels is about 3.2 arc min.

Poignant to us are the observations of March 1942 (entry 10). On the 2nd, Joe Hickox observed Mt. Wilson region 7378 and found places where the field was 4800 and 5900 G. Picture Hickox, working at a virtually deserted observatory, deserted because most of the staff were on leave for war-related activities. In the valley below bombers were being built for attacks on Germany. Approximately $15 \mathrm{~h}$ later, $\mathrm{H}$. von Klüber's assistant, a Dr. Haffner, observed the same sunspot. His maximum field was $4300 \mathrm{G}$; perhaps the region had evolved. This was in Potsdam on the outskirts of Berlin. Some of those Southern-California bombers were later to destroy large sections of Potsdam. Hickox was likely oblivious to these events and von Klüber 
never hints at anything untoward in his excellent post-war paper on the topic (von Klüber, 1947). (Also ironic, Potsdam was the birthplace of Martin Schwarzschild who, during the war, was decorated as a U.S. Army officer specializing in bombing surveys. Even more ironic, in May 1943, the designer of the solar tower telescope in Potsdam, Erich Mendelsohn, designed for the U.S. Army copies of typical Berlin tenements that were used for bombing tests in the desert of Utah.)

The record high field strength sunspot group (entry 10) prompted a comment by the Mt. Wilson staff (Anonymous, 1942). It was reported that both major umbrae of the group were darker than normal. This group produced a large flare and a subsequent "great" geomagnetic storm (Newton, 1942). It was also the source of the discovery of solar radio emission using meter-wave radar receivers, a discovery that was kept secret until after the war (Ellison, 1955).

Around 1978, the Mt. Wilson sunspot drawings improved, measurements were more frequent and some are works of art as well as science. However, because of inadequate calibration and limited range of the tipping plate micrometer, recent Mt. Wilson observers never reported fields much in excess of $3 \mathrm{kG}$ (before our corrections).

To see if there is a particular kind of sunspot that gives rise to strong fields, we have sought white light images for some of the entries in Table III. For this, we searched the U.S. Naval Observatory (USNO) archives, which now reside at Kitt Peak. These date from 1898 to 1974. Because Washington, D.C. is not a good seeing site, the USNO contracted in the 1930s with Mt. Wilson to make two exposures daily and send one to Washington. NSO now archives 9400 USNO and $5440 \mathrm{Mt}$. Wilson plates. The images we could readily find are shown in Figure 2. White light images of the spots observed by Livingston and Harvey have been previously published (Livingston, 1974; Harvey, 1986).

We note in Figure 2 that strong fields are found in both simple and complicated sunspot structures. While compiling Table III, we noted that most strong-field spots were found in large, complicated groups, often with light bridges - but we emphasize that this is not always the case. In other words, many complicated, large groups do not show unusually large fields and some simple spots do contain unusually strong fields. Evidently, the physical process that creates unusually strong fields is not closely related to the overall morphology of the sunspot group.

\section{Statistics}

A histogram of the strongest measured fields in 12804 sunspot groups from 1917 through 2004 is shown in Figure 3. Values less than $1000 \mathrm{G}$ being unreliable are not plotted. The full line is a combination of Mt. Wilson and Rome visual measurements from 1917 through 1974 plus entries 49 - 55 from Table III. In a few pre-1975 cases, the values were changed if another observatory reported a stronger field. In this set of data, the percentages of groups with strongest fields $>3 \mathrm{kG}$ is $4.6 \%$; $>3.5 \mathrm{kG}$, 


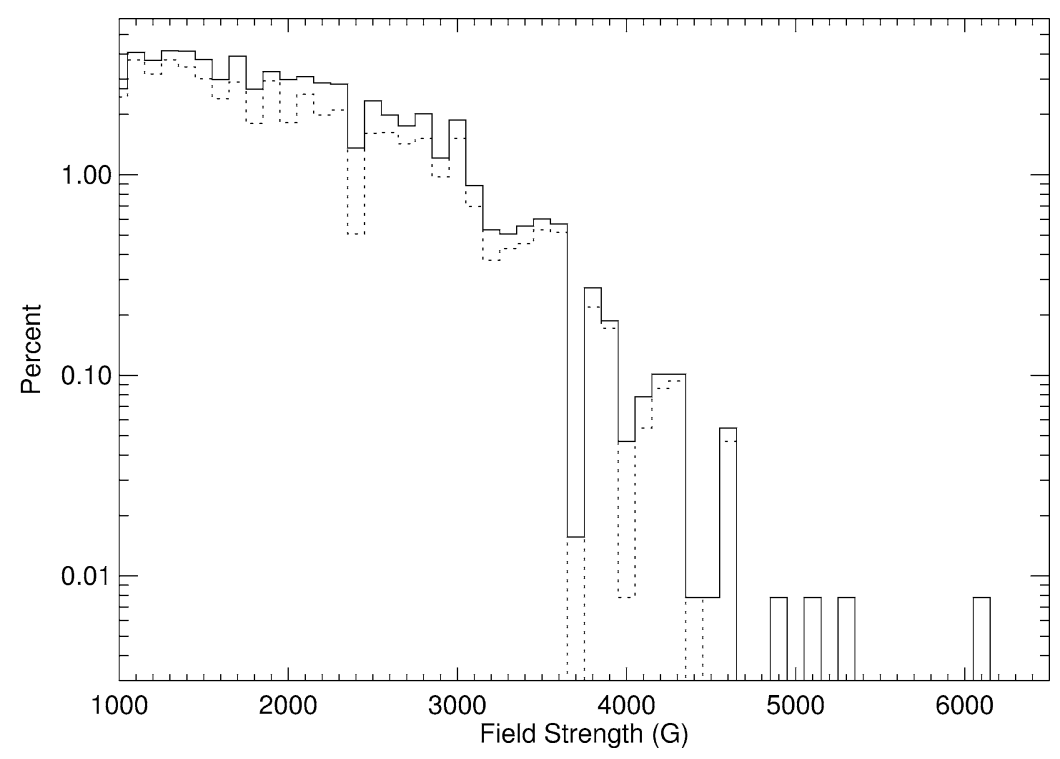

Figure 3. Distribution of maximum field strengths in 12804 sunspot groups measured at Mt. Wilson (1917 - 1964) and Rome (1965 - 1974). Seven measurements from Table III not included in the Mt. Wilson and Rome data were added. The dashed histogram is only Mt. Wilson measurements.

$1.5 \%$; $>4 \mathrm{kG}, 0.4 \%$ and $>4.5 \mathrm{kG}, 0.09 \%$. The dashed line represents $11051 \mathrm{Mt}$. Wilson visual measurements alone (corrected above $3600 \mathrm{G}$ according to Table II) and shows some structure that is hidden in the combined data set. One oddity is a relative dearth of $2400 \mathrm{G}$ values. Above this value, there is an excess of even values such as 2800 and $3000 \mathrm{G}$ versus odd values such as $2900 \mathrm{G}$. Below $2400 \mathrm{G}$, the situation is reversed. The odd-even behavior was noted in previous studies of the measurements (e.g. Houtgast and van Sluiters, 1948). We think that these departures from smooth behavior are explained in part by the ad hoc correction of measured fields developed by Hale and Nicholson as noted earlier. If the observers tended to use even rather than odd-numbered degree values, the odd-even asymmetry and its reversal at $2400 \mathrm{G}$ could also be explained. The low numbers of Mt. Wilson measurements at 3700,4000 , and $4400 \mathrm{G}$ are simply a result of coarseness of the corrections we applied, as listed in Table II.

With the exception of the record $6100 \mathrm{G}$ measurement, the distribution of field strengths between 3000 and $5300 \mathrm{G}$ in Figure 3 follows a power law with a steep slope of -9.5 fairly well. The failure of the $6100 \mathrm{G}$ measurement to follow such a fit suggests that it was either an incorrect measurement (unlikely since the field was measured as $5900 \mathrm{G}, 2$ days later) or a very rare event. One might well argue that the underlying distribution of maximum sunspot field strengths should be lognormal since that is the distribution that best represents maximum sunspot umbral areas (Baumann and Solanki, 2005). We cannot easily test that idea since 

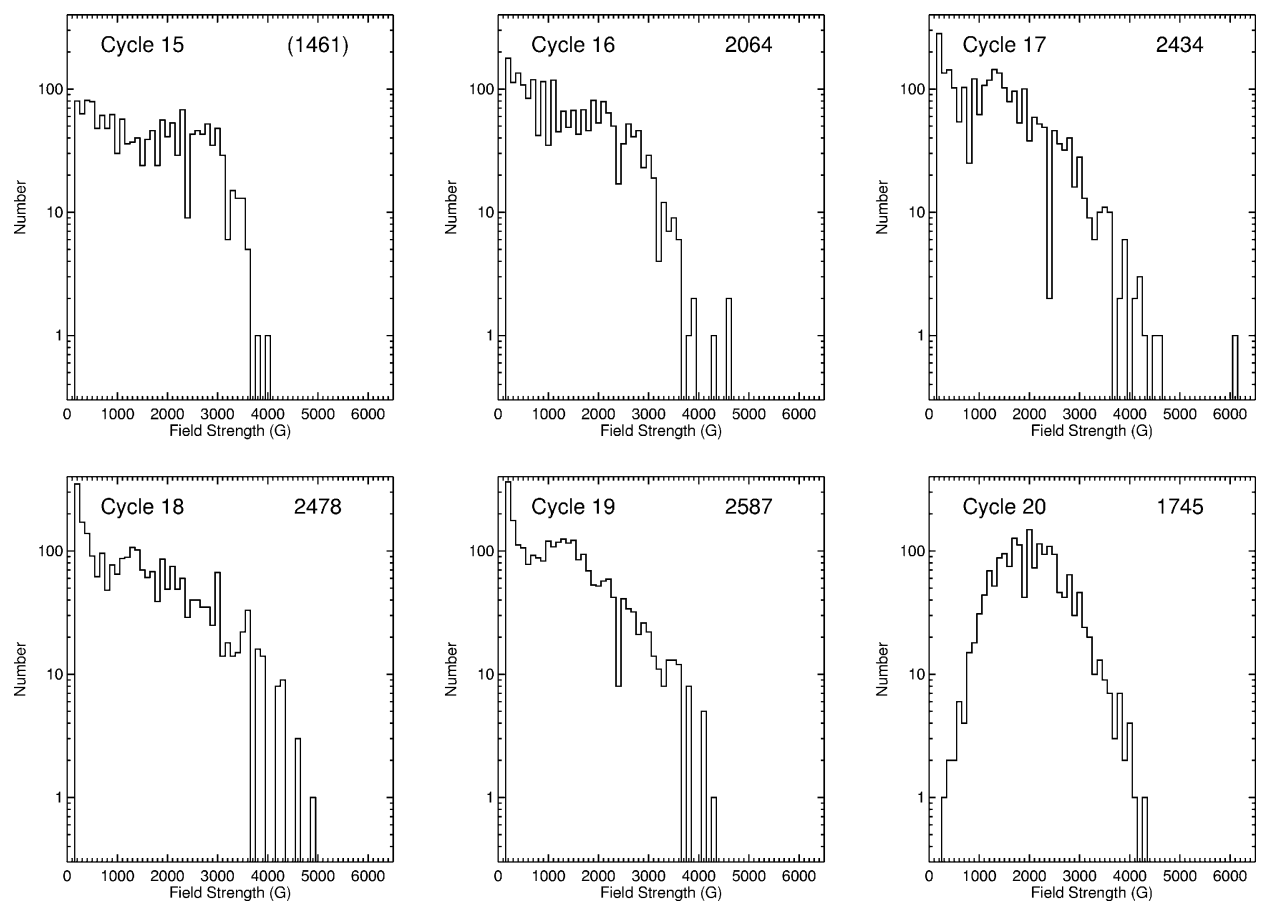

Figure 4. Distributions of measurements of maximum field strengths in 12769 sunspot groups by sunspot cycle number.

the measurements at low field strengths are seriously compromised as discussed in Section 2. We do note that a lognormal distribution of field strengths would make the $6100 \mathrm{G}$ measurement even more anomalous. The main point we want to make is that there is little evidence for more than one population of sunspot field strengths in the data set. If this is true, field strengths larger than $6 \mathrm{kG}$ must be very rare events indeed.

Figure 4 shows histograms of the measurements organized by sunspot cycle number. The number of measured groups is shown in each panel. Cycle 15 is incomplete and the latter part of cycle 19 was compromised by changes in observing technique at Mt. Wilson, which made strong fields hard to measure. Cycle 20 is entirely based on Rome data and looks very much like a lognormal distribution, though we know from modern observations (e.g. Livingston, 2002) that it is unlikely that any sunspots have field strengths less than about $1500 \mathrm{G}$. This lower limit became observationally obvious to one of us (LW) when a new grating was installed at Mt. Wilson in 1982.

We now consider whether strong-field sunspots are distributed any differently in time or latitude compared to all sunspot groups. To do this, we used a different data set. Namely, a listing of all 32122 sunspot groups observed at Mt. Wilson from 1915 to 2004 compiled by T. Shieber through mid-2002, which is located in the Mt. 


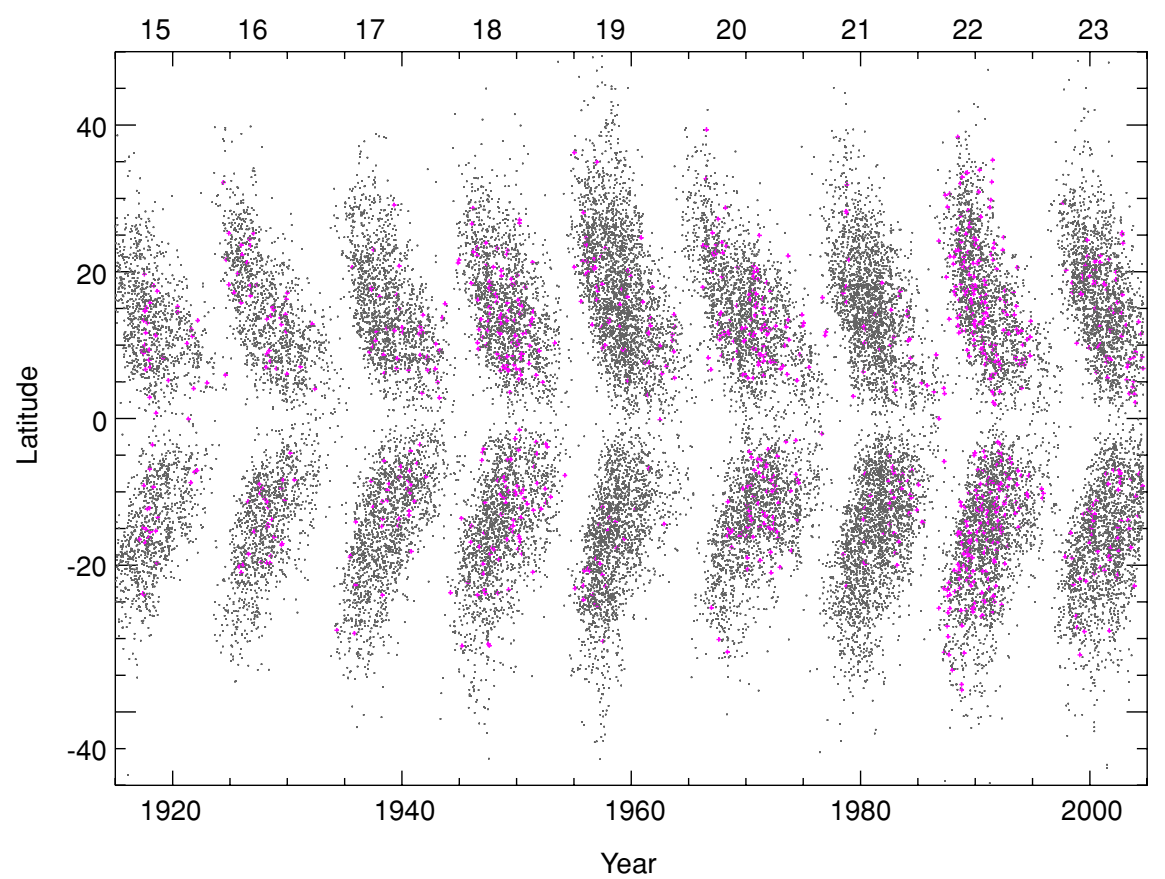

Figure 5. Time-latitude distribution of sunspot groups observed at Mt. Wilson. Magenta symbols mark groups with a field strength $>3 \mathrm{kG}$. Cycle numbers are above the plot.

Wilson archives. We extended this list through August 2004 and added measurements for 1917. No field strength values appear to be available for 1915 and 1916. Many of the groups included are small and have no field strength measurements but essentially all large groups have field strength measurements. In this data set, the maximum magnetic field strength for a sunspot group is listed only as an integer from 0 to 9 . The steps are nominally $500 \mathrm{G}$ with 1 representing $100-500 \mathrm{G}$ values.

Figure 5 is a butterfly diagram constructed from this data set. Each sunspot group is represented by a point. Latitudes were recorded to the nearest degree and to improve visibility we randomly dithered the measured latitude by up to half a degree. From 1917 to 1961, magenta symbols mark groups with field strength classes of 7 or greater (i.e. $>3 \mathrm{kG}$ ) and after 1961 for classes of 6 or greater. This change is required by the reduced sensitivity of Mt. Wilson measurements to strong fields after 1961 and results in a more homogeneous overall treatment.

Two interesting features are seen in Figure 5. First, the total number of sunspot groups in an odd-numbered cycle appears to be more than the number in the preceding even-numbered cycle. We checked this by counting the sunspot groups in the Mt. Wilson list and also in the Greenwich Photoheliograph Results listings for 1874 through 1976. Attempts to estimate the count for cycle 23 were not successful due to changes in the way sunspot groups are presently counted. The Greenwich 
results confirm that the number of groups in an odd cycle is about $132 \%$ of the number in the preceding even cycle (cycles $12-19$ ). Note that Greenwich counts groups that are visible on at least 2 days. Mt. Wilson counts groups that are visible on one or more days. The number of groups in odd cycles observed at Mt. Wilson is about $128 \%$ of the preceding even cycle (cycles 16-22). This odd-even imbalance is well known in the sunspot number time series and has been extensively discussed in that context (e.g. Mursula, Usoskin, and Kovaltsov, 2001). We note that despite changes in the way sunspot groups are counted, it appears that cycle 23 is anomalous and will not follow the odd-even pattern observed for at least the previous 17 cycles. That cycle 23 is anomalous in other ways has been discussed by de Toma et al. (2004).

Second, the number of strong-field sunspot groups also appears to exhibit an opposite odd-even cycle pattern. With the exception of cycles 16 and 17, which had nearly equal numbers of $>3 \mathrm{kG}$ field sunspot groups, later odd-numbered cycles have about $37 \%$ of the number of strong-field groups as their preceding evennumbered cycle (cycles 18-23). This result, demonstrated here for several solar cycles, was suggested by Bell (1959) for cycle 19 (based on Mt. Wilson data) and by Livingston (2002) for cycle 23 (based on Kitt Peak data). Expressed in another way, the average number of $>3 \mathrm{kG}$ sunspot groups in even-numbered cycles is 187 and in odd-numbered cycles 76 or about $60 \%$ less (cycles $15-23$ ). To see the effect in more detail, in Figure 6, we show the ratios of histograms for cycles 16-19 to
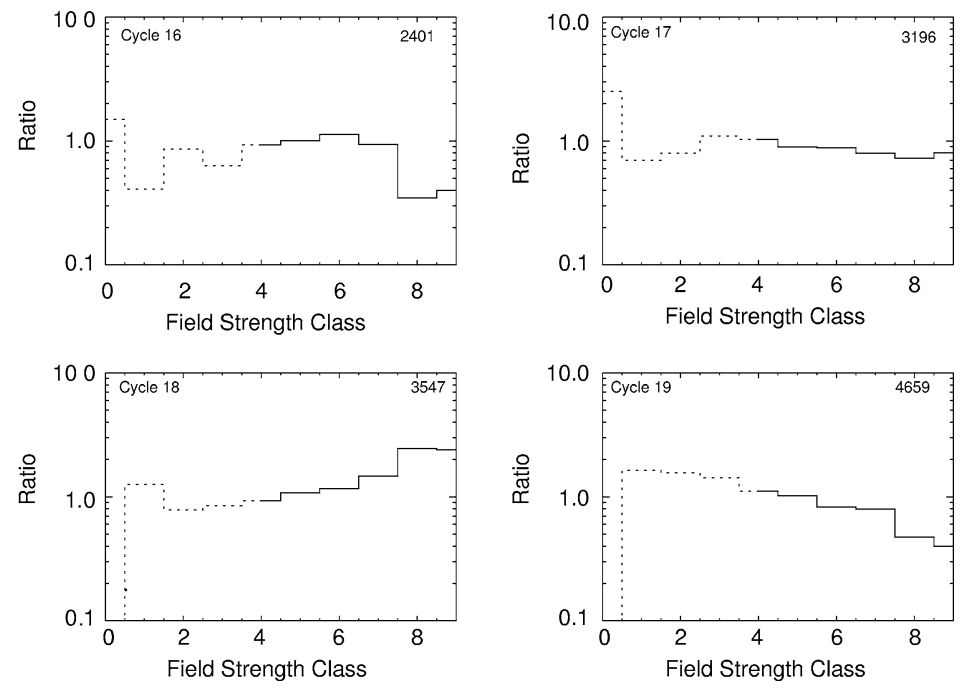

Figure 6. The ratio of the numbers of sunspot groups assigned to field strength classes compared to the average of cycles 16-19. The dashed line indicates less reliable low field strength classes. The numbers of groups in each cycle are indicated. The field strength classes and nominal strengths are: 1 , $100-500 \mathrm{G} ; 2,600-1000 \mathrm{G} ; 3,1100-1500 \mathrm{G} ; 4,1600-2000 \mathrm{G} ; 5,2100-2500 \mathrm{G} ; 6,2600-3000$ $\mathrm{G} ; 7,3100-3500 \mathrm{G} ; 8,3600-4000 \mathrm{G} ; 9$, > $4000 \mathrm{G}$. 

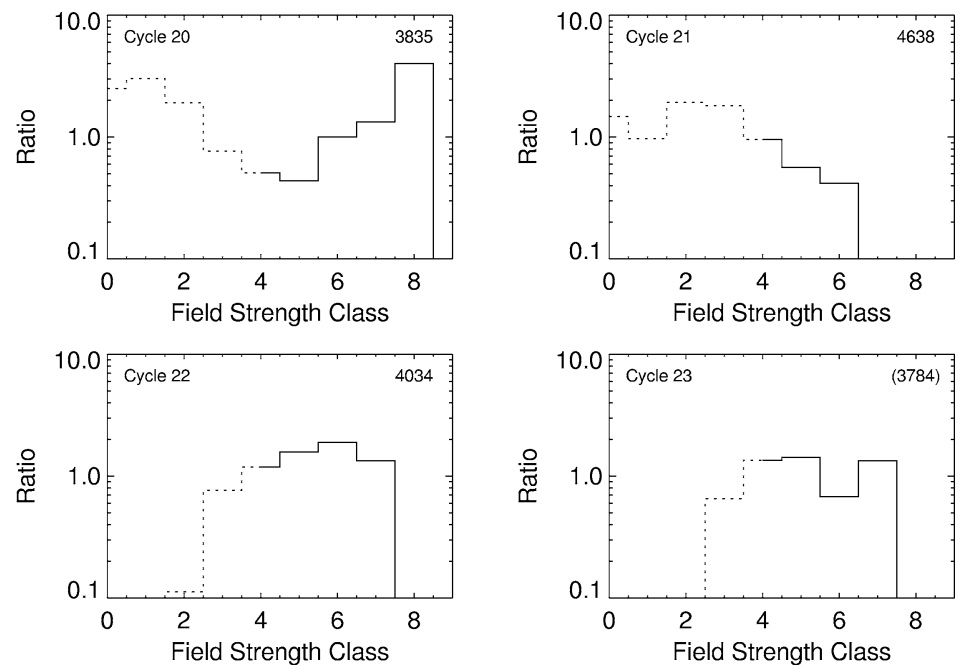

Figure 7. The ratio of the numbers of sunspot groups assigned to field strength classes compared to the average of cycles $20-23$. The dashed lines indicate less reliable low field strength classes. The numbers of groups in each cycle are indicated. Cycle 23 is truncated at August 2004. Due to observing changes, about one class should be added here to compare with the data shown in Figure 6.

the mean histogram for these cycles and in Figure 7 the same for cycles $20-23$. The cycles were grouped this way because of the major change in Mt. Wilson observing technique near the end of cycle 19. In both figures, for even-numbered cycles, we see a generally positive slope for classes of $>3$ and a negative slope for odd-numbered cycles. Exceptions are cycles 16 and 23.

When we first compared the butterfly diagrams of strong-field sunspot groups and all groups, we calculated the mean times and latitudes (and their variances) of each cycle in each hemispheres. Except for a time shift of about 150 days earlier in cycle 22 the timing of strong and all spot groups is indistinguishable. This simple analysis also showed no significant shift in the mean latitudes of strong and all sunspot groups.

Although Figure 5 suggests that there is no systematic difference between the time-latitude distributions of strong-field sunspot groups and all groups, stimulated by the work of Norton and Gilman (2004) we looked more carefully for any difference. Using umbral intensity measurements from MDI as a proxy for magnetic field strength (Norton and Gilman, 2004) grouped more than 600 umbral measurements into two brightness ranges. They found that about $64 \%$ of the time brighter umbras were poleward of darker umbras when both were present during a Carrington rotation. Figure 8 shows the annual average latitude of sunspot groups according to magnetic field strength class using the same data as for Figure 5. This plot shows no obvious systematic dependence of the average latitude of sunspot groups on their strongest magnetic field strengths. We then used our data to count how many sunspot groups in a given magnetic class appeared equatorward and poleward of 


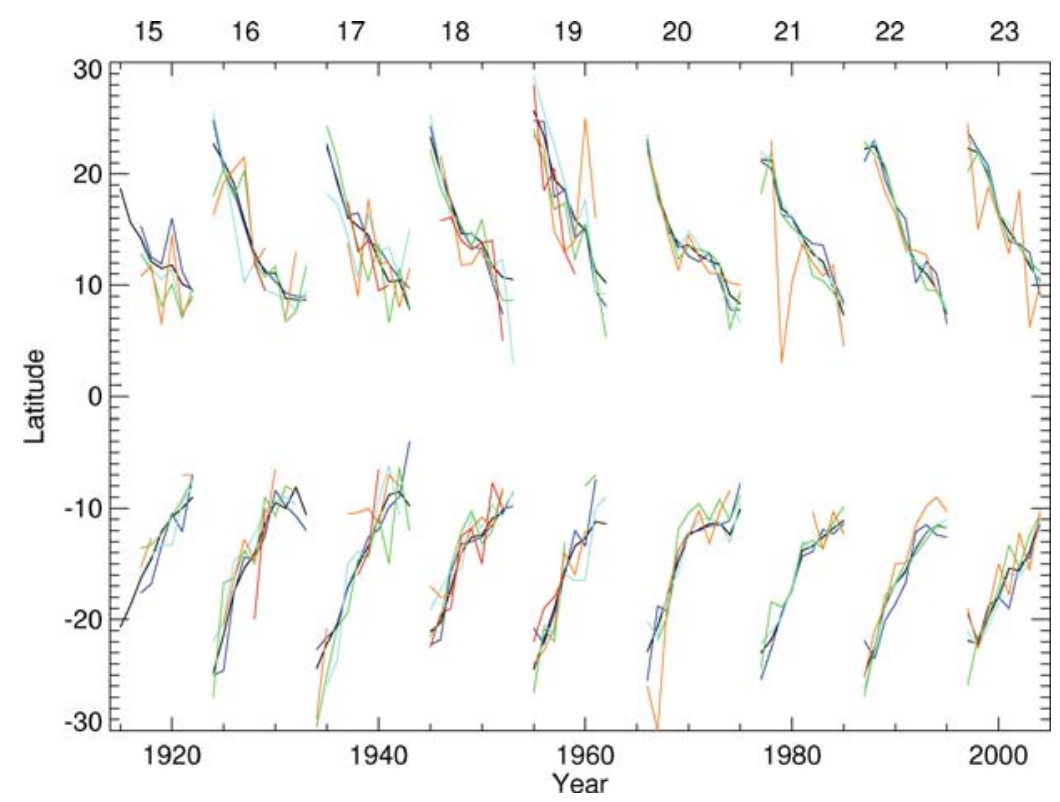

Figure 8. The average latitude by year and hemisphere of sunspot groups does not show any obvious systematic dependence on magnetic field strength. Colors indicate magnetic class. Black is all sunspot groups, blue is $1600-2000 \mathrm{G}$, cyan is $2100-2500 \mathrm{G}$, green is $2600-3000 \mathrm{G}$, orange is $3100-3500$ $\mathrm{G}$, and red is $3600-4000 \mathrm{G}$. Due to observing changes, the magnetic class was increased by one for data after 1962.

the average latitude of all groups on a year-by-year basis for cycles $15-23$. We incremented the magnetic class by one for observations after cycle 19. For 5605 groups with fields greater than $2500 \mathrm{G}, 57.24 \%$ appeared closer to the equator than the average latitude of all groups on an annual basis. Thus, similar to Norton and Gilman, we find a slight tendency for stronger field sunspot groups to be equatorward of all sunspot groups. The effect is small, being less than a $1^{\circ}$ shift of an underlying distribution which is 10 or more degrees wide. What little effect we see could be an observational bias. For example, since strong-field sunspot groups are typically large and complex, it is conceivable that observers systematically assign an average latitude to the group which is slightly biased to the equator.

\section{The Upper Limit of Observed Field Strength}

Here, we compare our results with some current ideas about the physics of individual sunspots (see reviews by Jahn, 1992, 1997; Solanki, 2003; Solanki, Inhester, and Schüssler, 2006; and references therein). First, we discuss strong umbral fields and then strong fields in light bridges. Unfortunately, sunspots are complicated and there is no comprehensive model to compare with our observations. Therefore, much of this section is not as rigorous as we would like. With that caveat, we consider 
our results and several basic ideas about the physics of sunspots. In particular, the umbral magnetic field strength is widely thought to be governed by a horizontal force balance with external gas pressure plus the height variation of tension along the curved field lines. Forces caused by mass motions are generally thought to be insignificant (Maltby, 1977). The level we see in an umbra is considerably deeper than in the quiet Sun (i.e., the Wilson depression) as a result of lower temperature and density in the umbra. In this scenario, a greater field strength produces more cooling and a deeper Wilson depression. Are these ideas consistent with our observations or are additional factors needed?

In an over-simplified magnetohydrostatic model of a sunspot with an umbral field strength that varies with depth but is constant in a horizontal plane (e.g. Maltby, 1977) the ratio of umbral to photospheric temperature should be proportional to $1-\left(B^{2} / P_{o}\right)$, where $P_{o}$ is the external gas pressure at a depth corresponding to the umbra. Guided by this result, we used Livingston's (2002) continuing $1564.8 \mathrm{~nm}$ observations of umbral to photospheric intensity ratio and magnetic field strength and converted the ratios to brightness temperatures assuming that the photospheric brightness temperature at that wavelength is $6700 \mathrm{~K}$. Limiting ourselves to umbral brightness temperatures between 5300 and $4600 \mathrm{~K}$ (in order to keep $P_{o}$ approximately constant), we fit the temperatures of 232 umbras with a linear function of $B^{2}$. A higher order function did not significantly improve the fit over the measured field strength range of $2250-3550 \mathrm{G}$. We extrapolated the resulting fit to field strengths of 4,5 , and $6 \mathrm{kG}$ and got temperatures of 4460,3740 , and $2860 \mathrm{~K}$, respectively. Bearing in mind the unrealistic physics and limited observational ranges underlying this extrapolation, we speculate that a limit on the field strength of a very cold umbra might be set by heat invading from the spot's hot surroundings which would tend to raise gas pressure, expand the field and thereby limit its strength. In other words, the umbral magnetic field strength may be limited by an inability of the field to reduce upward convective heat flux from beneath the spot enough to sustain an even stronger field. While this speculative scenario is consistent with the idea that the umbral magnetic field becomes fragmented at a depth not far below the visible layers, a more monolithic field structure cannot be ruled out by our results. Perhaps the degree of fragmentation of the umbral field is important in controlling the maximum field strength.

Are very strong umbral fields associated with inferred Wilson depression depths that are consistent with available observations? We first recall that measurements of Wilson depressions are difficult and not always consistent (e.g. Balthasar and Wöhl, 1983; Collados, del Toro Iniesta, and Vazquez, 1987; Bagare and Gupta, 1998; Hudson et al., 2006). The observed range of depths extends to $1 \mathrm{Mm}$ with $600 \mathrm{~km}$ as a typical value. As a crude comparison with our results, we imagine a flux tube in which the external gas pressure is balanced only by magnetic pressure and ask what field strengths are required to maintain balance as a function of depth. Using a model of the upper convection zone (Spruit, 1974), we find observationally acceptable depths of 230, 400, 550 and $670 \mathrm{~km}$ for uniform field strengths of 3, 
4,5 , and $6 \mathrm{kG}$, respectively. Of course, this comparison neglects the non-zero gas pressure in sunspot umbras and the additional horizontal force of the depth variation of field line curvature.

Adding umbral gas pressure to the pressure balance reduces the field strength required at a given depth so the Wilson depression for a given field strength will increase. We followed Maltby (1977) and placed an empirical model of a dark sunspot umbra (model E of Maltby et al., 1986) at various assumed Wilson depression depths relative to the convection zone model. For example, with a Wilson depression depth of $600 \mathrm{~km}$, optical depth 0.1 is at $510 \mathrm{~km}$ and the pressure difference there between the exterior and umbral models is $8 \times 10^{5} \mathrm{dyn} \mathrm{cm}^{-2}$, which is balanced by a magnetic field strength of about $4500 \mathrm{G}$. So including the gas pressure increases the depths needed to balance $3-6 \mathrm{kG}$ umbral magnetic fields by only about $60-20 \mathrm{~km}$, respectively. The modeled Wilson depressions still remain consistent with available measurements.

Similarly, adding the effect of the depth variation of field line curvature to the pressure balance requires a further increase in the Wilson depression for a given field strength. It is uncertain how important field line curvature is to the force balance in a sunspot umbra. A fair guess for the strong part of an umbral field where the field is predominantly vertical might be an additional 50\% of the pressure due to the field alone (but see Mattig, 1969). Using this estimate, Wilson depressions would be increased by $110-160 \mathrm{~km}$ for field strengths of 3-6 kG. Including this increase, the Wilson depression for a $6 \mathrm{kG}$ field is $850 \mathrm{~km}$, which is approaching the largest observed depths. Thus, our crude estimates of the depth of the Wilson depression associated with the strongest observed fields are not contradicted by available observations.

Although we did not include information about the disk location of the spots with strong magnetic fields, our impression from compiling Table III is that they were observed mainly near the disk center with a few notable exceptions near the limb. The umbral atmosphere is rather transparent but near the limb we would still expect to observe higher levels with smaller field strengths. To account for strong fields near the limb, we suggest that these observations refer to areas in umbras and adjacent light bridges where the field is predominantly horizontal.

In contrast to umbras and light bridges with mainly vertical magnetic fields, there is almost no work on umbras and light bridges with strong horizontal fields. Tanaka (1991) and Zirin and Wang (1993) have shown clear observations that such fields exist. Several of the spots in Table III are likely to be of this type. Such fields are confined to $\delta$-type sunspot groups. These groups often appear to be formed by the $0.1-0.5 \mathrm{~km} \mathrm{~s}^{-1}$ motion of opposite polarity sunspots toward each other. As pointed out by Tanaka, the dynamic pressure of this motion is negligible compared to the magnetic pressure unless the motion involves densities found quite deep in the convection zone. It is possible that local flows many times faster than the proper motion of the spots might be involved in a force balance. However, available observations suggest that flows in light bridges are no more than 10 times faster 
than the proper motion of sunspots and this is not likely to affect the force balance. Given the present poor knowledge about these strong horizontal fields, there is little that we can add except to emphasize that they exist and deserve more study.

\section{On the Spatial and Temporal Distributions of Strong-Field Sunspots}

Here, we discuss two of our main results: the similarity within a given solar cycle of the time and latitude distributions of strong-field and all sunspot groups, and the odd-even cycle variation of the number of strong-field groups. As in the previous section, we are again hampered by a lack of detailed physical models. Specifically needed are models of flux tube formation and how they rise through the convection zone and emerge at the surface. So, again our discussions are over-simplified and speculative.

According to current ideas of the rise of magnetic flux from the base of the convection zone to form active regions, Coriolis force tends to make the flux rise parallel to the rotation axis. Since sunspots are not observed at high latitudes, flux must rise through the convection zone rapidly enough to avoid much influence by the Coriolis force. This is possible only if the buoyancy force is strong, in other words, the field strength of the rising flux is large $(\sim 100 \mathrm{kG})$. The greater the field strength, the more nearly radially the flux moves. In this scenario, we expect stronger magnetic fields to appear at lower latitudes. We find only slight observational evidence for this. Evidently, a strong-field sunspot retains only a faint signature of its original strength at the base of the convection zone. This would be the case if the observed surface field strength is dominated by nearsurface processes and the spot is disconnected from its deep roots. We note that the spots with strong fields all appear to be near a stage of maximum development and that the duration of strong fields lasts a few days. Schüssler and Rempel (2005) suggest that disconnection occurs a few days after the appearance of a spot as a result of pressure increases due to gas flows from above and below to a level a few $\mathrm{Mm}$ below the sunspot. This upsets a force balance between the field and the surrounding medium, which results in explosive fragmentation of the field and a disconnection.

In contradiction to the previous paragraph, our finding of an odd-even cycle alternation of the number of strong-field groups in the ratio of 4:10 that is out of phase with the number of all sunspot groups seems to require that the field strength does retain a signature of processes at the base of the convection zone. Less likely, near-surface effects would have to change from cycle to cycle and it is hard to imagine how such local conditions would be sensitive to a cycle's odd or even parity. Concentrating on conditions at the base of the convection zone, we need a dynamo that produces a larger number of sunspot groups in one cycle and in the next cycle fewer total groups but more strong-field groups - in both hemispheres simultaneously. One speculation is that one cycle produces flux ropes that are more resistant to shredding in that cycle than in the next (Schüssler, 1980). This 
would result in fewer, stronger spots. Another notion is that there is a weak fossil magnetic field in the radiative interior that interacts with the dynamo to produce cycle parity differences (cf. Rüdiger and Kitchatinov, 1997; Gough and McIntyre, 1998; Mursula, Usoskin, and Kovaltsov, 2001). Ideas like these are pure speculation until we have a better notion of how magnetic flux is processed at the base of the convection zone. Perhaps helicity is involved.

\section{Conclusion}

We examined a 9-decade record of measurements of the maximum magnetic field strengths of sunspot groups. The distribution of strongest fields follows a power law (with a steep exponent of about -9.5 for strengths above $3 \mathrm{kG}$ ) and appears to be drawn from a single population. This means that strong-field sunspots are either rare or hard to observe. We found 55 groups out of about 32000 observed groups $(0.2 \%)$ with plausible measurements of $4 \mathrm{kG}$ or more. The record measurement of $6100 \mathrm{G}$ is probably an exceedingly rare occurrence.

Strong fields may occur in simple sunspots but more frequently occur in large, complex groups, often in connection with light bridges of $\delta$-type spots. Strong fields are observed for up to a few days near the maximum phase of development of a sunspot group. Field strengths up to $6 \mathrm{kG}$ are consistent with highly idealized magnetohydrostatic models of sunspot umbras. Stronger fields would require implausibly low temperatures and deep Wilson depressions.

We found at best only a slight equatorward preference of the latitude distribution of strong-field groups compared to all sunspot groups. This suggests that the maximum field strengths are set by near-surface processes and do not convey much information about conditions deep inside the sun.

On the other hand, we found that, on average, the number of strong-field sunspot groups is $60 \%$ smaller in odd-numbered cycles compared to the preceding evennumbered cycle while the number of all groups is about $30 \%$ larger. It seems likely that this alternation is due to some unknown process near the base of the convection zone that is conveyed to the surface.

In future work, it would be useful to better incorporate the large number of measurements of sunspot field strengths made in the former Soviet Union. There are also available hundreds of thousands of measurements of individual sunspots from Mt. Wilson, Rome, and Potsdam that have not been studied. Many questions remain that we have not addressed in this survey. For example: What is the time evolution of the field strength in the strong-field spots? What center-to-limb variation exists in the distribution of strong-field measurements? What is the distribution of strongfield spots among various spot morphologies? To what extent does the solar cycle conserve total flux but distribute it differently between weak and strong fields? These questions and more could be addressed with the available records. To that end as time permits, one of us (LW) is scanning the original Mt. Wilson drawings 
and placing them on the Internet at ftp://howard.astro.ucla.edu/pub/obs/drawings. It is unfortunate that regular observations optimized to measure sunspot magnetic field strengths are no longer being made anywhere. Spectropolarimetric instruments could do this task with appropriate analysis algorithms.

\section{Acknowledgments}

This study includes data from the synoptic program at the 150-Foot Solar Tower of the Mt. Wilson Observatory. The Mt. Wilson 150-Foot Solar Tower is operated by UCLA, with funding from NASA, ONR and NSF, under agreement with the Mt. Wilson Institute. We thank Robert Howard for donation of a bound copy of Mt. Wilson sunspot data. We also thank the Solar Physics Research Corporation for loan of a set of microfilm copies of the Mt. Wilson sunspot drawings. V. Lozitsky and N. Lozitska kindly provided a list of strong-field measurements made in the former Soviet Union. Some of these measurements are included in Table III. We thank N. Stepanian for loan of the original plate reproduced in Figure 1. We also thank A. Norton for carefully reading a draft of this paper and for stimulating discussions. We thank the referee, S. Solanki, for thoughtful and valuable suggestions. The National Solar Observatory is operated by the Association of Universities for Research in Astronomy, Inc., under cooperative agreement with the National Science Foundation.

\section{References}

Anonymous: 1936, Publ. Astron. Soc. Pac. 48, 124.

Anonymous: 1942, Publ. Astron. Soc. Pac. 54, 110.

Anonymous: 1958, Carnegie Inst. Washington Yearbook, Vol. 58, p. 43.

Anonymous: 1962, Carnegie Inst. Washington Yearbook, Vol. 62, p. 6.

Bagare, S.P. and Gupta, S.S.: 1998, Bull. Astron. Soc. India 26, 197.

Balthasar, H. and Wöhl, H.: 1983, Solar Phys. 88, 71.

Baranovsky, E.A. and Petrova, N.N.: 1957, Sol. Dann. (5), 118.

Baumann, I. and Solanki, S.: 2005, Astron. Astrophys. 443, 1061.

Bell, B.: 1959, Publ. Astron. Soc. Pac. 71, 165.

Bell, B. and Glazer, H.: 1958, Smithsonian Contr. Astrophys. 2, 161.

Bell, B. and Glazer, H.: 1959, Smithsonian Contr. Astrophys. 3, 25.

Brants, J.J. and Zwann, C.: 1982, Solar Phys. 80, 251.

Cimino, M.: 1965 - 1975, Solar Phenom. Monthly Bull., Osserv. Astron. Roma, Nos. 85-212.

Collados, M., del Toro Iniesta, J.C., and Vazquez, M.: 1987, Solar Phys. 112, 281.

Cowling, T.G.: 1946, Mon. Not Roy. Astron. Soc. 106, 218.

Croce, V.: 1967, Mem. Soc. Astron. Ital. 38, 215.

de Toma, G., White, O.R., Chapman, G.A., Walton, S.R., Preminger, D.G., and Cookson, A.M.: 2004, Astrophys. J. 609, 1140.

Ellerman, F.: 1919, Publ. Astron. Soc. Pac. 31, 16.

Ellison, M.A.: 1955, The Sun and its Influence, Macmillan, New York.

Gough, D.O. and McIntyre, M.E.: 1998, Nature 394, 755. 
Grotrian, W.: 1953, Publ. Astrophys. Obs. Potsdam 29(4).

Hale, G.E. and Nicholson, S.B.: 1938, Magnetic Observations of Sunspots 1917-1924, Carnegie Inst. Washington, Washington, D.C.

Hale, G.E., Ellerman, F., Nicholson, S.B., and Joy, A.H.: 1919, Astrophys. J. 49, 153.

Harvey, J.: 1986, in W. Deinzer, M. Knölker and H.H. Voigt (eds.), Small Scale Magnetic Flux Concentrations in the Solar Photosphere, Vandenhoeck und Ruprecht, Göttingen, p. 25.

Hudson, H.S., Fivian, M.D., Wilson, B.M., and Zahid, H.J.: 2006, Bull. Am. Astron. Soc. 38, 230.

Houtgast, J. and van Sluiters, A.: 1948, Bull. Astron. Inst. Netherlands 10, 325.

Jahn, K.: 1992, in J.H. Thomas and N.O. Weiss (eds.), Sunspots: Theory and Observations, Kluwer Academic Publishers, Dordrecht, Holland, p. 139.

Jahn, K.: 1997, in B. Schmieder, J.C. del Toro Iniesta, and M. Vazquez (eds.), ASP Conf. Ser. 118, 122.

King, R.B.: 1934, Astrophys. J. 80, 136.

Kopp, G. and Rabin, D.: 1992, Solar Phys. 141, 253.

Livingston, W.C.: 1974, in Y. Nakagawa and D.M. Rust (eds.), Flare-Related Magnetic Field Dynamics, High Altitude Obs., Boulder, p. 269.

Livingston, W.C.: 1976, Solar Phys. 48, 196.

Livingston, W.C.: 2002, Solar Phys. 207, 41.

Lozitsky, V.G.: 1993, Kinematika i Fizika Nebesnykh Tel. 9(3), 23.

Lozitsky, V.G. and Staude, J.: 2003, ASP Conf. Ser. 307, 378.

Maltby, P.: 1977, Solar Phys. 55, 335.

Maltby, P., Avrett, E.H., Carlsson, M., Kjeldseth-Moe, O., Kurucz, R.L., and Loeser, R.: 1986, Astrophys. J. 306, 284.

Mattig, W.: 1969, Solar Phys. 8, 291.

Mursula, K., Usoskin, I.G., and Kovaltsov, G.A.: 2001, Solar Phys. 198, 51.

Newton, H.W.: 1942, Observatory 64, 260.

Nicholson, S.B.: 1925 - 1960, Publ. Astron. Soc. Pac. 37-72.

Nicholson, S.B.: 1933, Publ. Astron. Soc. Pac. 45, 51.

Norton, A.A. and Gilman, P.A.: 2004, Astrophys. J. 603, 348.

Richardson, R.S. and Schwarzschild, M.: 1950, Astrophys. J. 111, 351.

Ringes, T.S.: 1965, Astron. Nor. 10, 29.

Ringes, T.S. and Jensen, E.: 1960, Astron. Nor. 7, 99.

Rüdiger, G. and Kitchatinov, L.L.: 1997, Astron. Nachr. 318, 273.

Schüssler, M.: 1980, Nature 288, 150.

Schüssler, M. and Rempel, M.: 2005, Astron. Astrophys. 441, 337.

Shpitalnaya, A.A., Makarov, V.I., and Den, O.E.: 1973, Sol. Dann. (2), 73.

Solanki, S.K.: 2003, Astron. Astrophys. Rev. 11, 153.

Solanki, S.K., Inhester, B., and Schüssler, M.: 2006, Rep. Prog. Phys. 69, 563.

Spruit, H.C.: 1974, Solar Phys. 34, 277.

Steen, O. and Maltby, P.: 1959, Astrophys. Norv. 6, No. 10.

Stepanov, V.E., Shaposhinkova, E.F., and Petrova, N.N.: 1962, Ann. IGY 23, 1.

Steshenko, N.V.: 1967, Izvest. Crimean Astrophys. Obs. 37, 21.

Tanaka, K.: 1991, Solar Phys. 136, 133.

von Klüber, H.: 1947, Z. Astron. 24, 121.

von Klüber, H.: 1955, Vistas Astron. 1, 751.

Wallace, L. and Livingston, W.: 2005, An Atlas of Sunspot Umbral Spectra in the Visible from 15,000 to $25,000 \mathrm{~cm}^{-1}$ (3920 to $6664 \AA$ ), Revised. Tech. Report No. 05-001, National Solar Obs., Tucson.

Wallace, L., Hinkle, K., and Livingston, W.: 2001, Sunspot Umbral Spectra in the Region from 4000 to $8640 \mathrm{~cm}^{-1}$ (1.16 to $2.50 \mu \mathrm{m}$ ), Tech. Report No. 01-001, National Solar Obs., Tucson.

Zirin, H. and Wang, H.: 1993, Solar Phys. 144, 37. 